\section{Notch it up to the antibodies}

\section{By Tim Fulmer, Senior Writer}

Researchers from Roche's Genentech Inc. and Exelixis Inc., ${ }^{1}$ and a separate team at Aveo Pharmaceuticals Inc., have designed antibodies that are selective for cancer-associated subtypes of the Notch receptor. Both groups expect that hitting only the Notch receptors involved in cancer will offer improved safety compared with that for $\gamma$-secretase inhibitors and other molecules that target all Notch receptors.

There are four Notch receptorsNotch homolog 1 translocation-associated (NOTCH1), Notch homolog 2 (NOTCH2), NOTCH3 and NOTCH4-which are expressed on the cell surface and help regulate cell fate and proliferation during the development of healthy tissue. Notch signaling begins when a Notch receptor binds one of its ligands, which triggers a sequence of proteolytic cleavage events involving $\alpha$-secretase and $\gamma$-secretase that result in a clipped form of the receptor called Notch intracellular domain (NICD). That peptide then translocates to the nucleus to upregulate expression of genes that promote cell growth and proliferation (see Figure 1, "Notch-targeting antibodies").

In cancer, mutations in the Notch receptor can lead to activation of the pathway even in the absence of the ligand, resulting in overexpression of downstream genes that drive tumor growth. ${ }^{2}$

Although the pathway contains a wealth of potential targets, a key concern has been that a lack of selectivity could lead to toxic blockade of Notch signaling in healthy tissue. For example, $\gamma$-secretase inhibitors, which block signaling through all four Notch inhibitors, have been associated with GI toxicity in animals and cancer patients. ${ }^{3,4}$

Antibodies are a logical choice to precisely attack Notch because they can be designed to target only those receptors that are activated in a particular cancer. Small molecules are typically unable to accomplish that task because they target Notch pathway enzymes, which are active in both cancerous and healthy tissue.

Both the Genentech-Exelixis and the Aveo groups initially focused on developing antibodies specific for NOTCH1, which has been associated with multiple cancers and carries activating mutations in more than $50 \%$ of T cell acute lymphoblastic leukemias (T-ALLs). ${ }^{5}$

The Genentech-Exelixis team used phage display to generate a human NOTCH1 antibody that showed activity against a T-ALL cell line expressing an aberrantly activated NOTCH1. The antibody triggered cell-cycle arrest and reduced cell proliferation compared with control. In a mouse xenograft model of T-ALL, animals given the antibody had regression of established tumors compared with mice given a control antibody. In a mouse model of colon cancer, the NOTCH1 antibody slowed tumor growth compared with control.

Blocking NOTCH1 also produced less GI toxicity compared with inhibiting multiple Notch receptors. Mice receiving the NOTCH1 antibody maintained their weight and had lower levels of intestinal goblet cell metaplasia compared with mice that received two antibodies, one selective for NOTCH1 and the other for NOTCH2. Goblet cell metaplasia causes the severe diarrhea associated with inhibiting multiple Notch receptors.

"In reducing or avoiding the goblet cell metaplasia that is a hallmark of a general Notch block, our receptor-specific inhibitors represent a clear breakthrough over existing pan-Notch inhibitors such as $\gamma$-secretase inhibitors," corresponding author Christian Siebel and colleagues at Genentech and Exelixis wrote in their paper in Nature.

The NOTCH1-specific antibody designed by the Aveo team, dubbed AV-232, inhibited activation of NOTCH1 by all four of its ligands in vitro and also blocked expression of a panel of NOTCH1-dependent genes. AV-232 was well tolerated in mouse models and did not result in dose-limiting gut toxicity. The study was led by Aveo researcher Ronan O’Hagan.

Although both the Genentech antibody and AV-232 target NOTCH1, they are "very different beasts," according to Jeno Gyuris, SVP of research at Aveo. "The Aveo antibody targets the ligand-binding domain of NOTCH1, while the Genentech antibody targets a NOTCH1 domain that is cleaved by a cell surface ADAM protease following ligand binding."

Gyuris said it is unknown whether the downstream effects of the two antibodies might differ and potentially lead to the suppression of different cancer-related genes.

Aveo presented its data last week at the American Association for Cancer Research (AACR) meeting in Washington, D.C.

\section{Moving forward}

Genentech now has two main goals in its preclinical Notch program.

"First of all, we want to determine what cancers would most benefit from the NOTCH1 and NOTCH2 antibodies," Siebel told SciBX. "For the NOTCH1 antibody, T-ALL would be an obvious candidate, as would colon and breast cancer. For the NOTCH2 antibody, work by ourselves and others suggests that melanoma and B cell malignancies would benefit."

The Nature paper presented no in vivo data on the activity of the NOTCH2 antibody. Other groups have implicated NOTCH2 in malignant melanoma, B cell lymphomas and other cancers. ${ }^{6,7}$ 
Figure 1. Notch-targeting antibodies. Researchers from Roche's Genentech Inc. and Exelixis Inc., and a separate team at Aveo Pharmaceuticals Inc., have designed antibodies specific for Notch receptor subtypes. Both groups believe the specificity of their therapeutics will result in improved safety compared with $\gamma$-secretase inhibitors and other Notch-targeting molecules.

[a] At the cell surface, Notch receptor ligands such as Jagged and delta-like proteins bind to the transmembrane Notch receptor, of which there are four subtypes.

[b] Interactions between the Notch receptor and its ligand trigger a sequence of proteolytic cleavage events. On the extracellular side of the membrane, $\alpha$-secretase enzymes called ADAM metallopeptidase domain 17 (ADAM17; TACE) or ADAM10 cleave the Notch receptor and generate a substrate for the $\gamma$-secretase complex inside the cell. Next, the $\gamma$-secretase complex cleaves an intracellular portion of the Notch receptor, generating the Notch intracellular domain (NICD).

[c] NICD then translocates to the nucleus, where it interacts with transcription factors to upregulate gene expression. In cancer, NICD can induce the expression of oncogenes that can trigger tumor growth.

By targeting the cell surface Notch receptor, the antibodies are predicted to prevent pro-oncogenic Notch signaling. The antibodies reported by both research groups are designed to target only those Notch receptor subtypes that are associated with cancer, thus avoiding Notch blockade in healthy tissues, which can lead to severe GI toxicity.

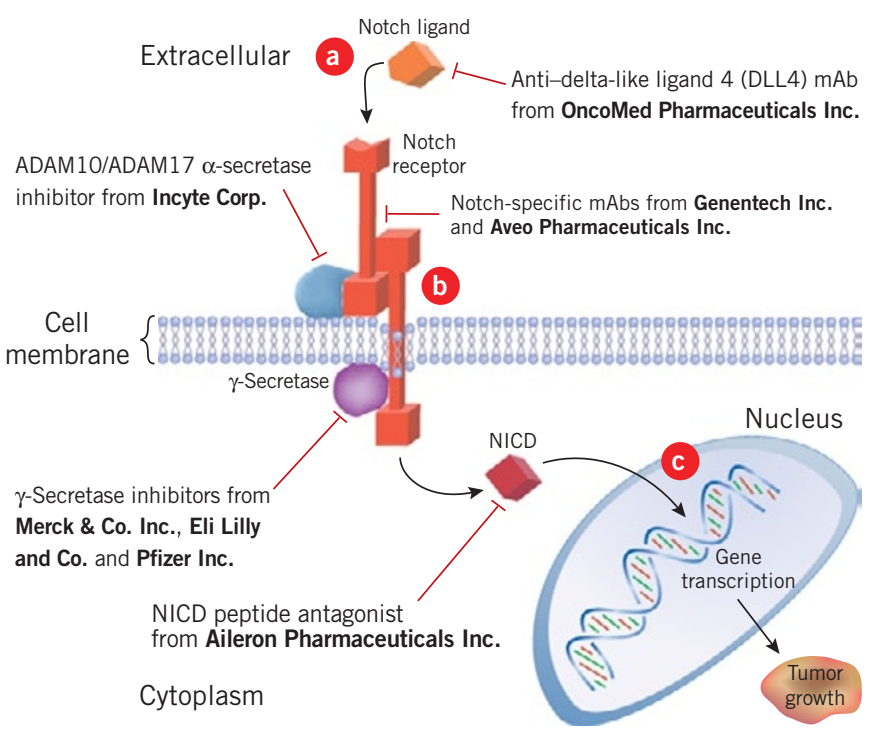

"Secondly," said Siebel, "we want to carry out mechanism-ofaction studies and look downstream of the Notch receptors to learn what genes are being turned on or turned off when we treat with our antibody. That information should help us understand, for example, whether selective blockade of Notch receptors is affecting general tumor growth pathways, or only cancer stem cell proliferation, or perhaps a combination of the two."

Siebel is a senior scientist in molecular biology at Genentech.

A first priority for Aveo is ongoing efforts to humanize AV-232, according to Gyuris. The company also has identified a NOTCH3specific antibody, which will be humanized in the near future. Like NOTCH1 and NOTCH2, NOTCH3 has been associated with specific cancers, including non-small cell lung cancer (NSCLC) and ovarian cancer. ${ }^{8,9}$

Although its three-year Notch deal with Genentech has run its course, Exelixis continues to interrogate the biology of the Notch pathway. "We have an ongoing collaboration with company cofounder Spyros Artavanis-Tsakonas, who developed a collection of transgenic mice that are being used to more fully elucidate the biology of the Notch pathway, particularly with respect to stem cell biology," said Michael Morrissey, president of R\&D.

Artavanis-Tsakonas is professor of cell biology at Harvard Medical School.

Under the 2005 deal, Genentech generated antibodies against Notch pathway targets provided by Exelixis. The resulting Notchselective antibodies are owned by Genentech, which is now responsible for manufacturing and clinical development, while Exelixis is eligible for milestones and royalties.

\section{Finding a niche}

Despite the positive data, other developers in the pathway argued the new antibodies are unlikely to crowd out the other approaches to inhibiting Notch. Indeed, it sometimes can be necessary to target multiple Notch receptors to get a therapeutic effect. Also, clinicians have been able to modify the dosing regimens of pan-Notch blockade to lower toxicity without impairing efficacy.

In some cancers, antibodies that are specific for Notch subtypes may be too selective to adequately treat the disease. "Certain tumor types may have multiple Notch pathway defects in multiple tumor compartments and may therefore benefit more from a pan-Notch inhibitor approach" such as targeting the $\gamma$-secretase complex, said Thomas Cirrito, director of operations at Stemline Therapeutics Inc.

Stemline has two $\gamma$-secretase inhibitors-SL-301 and SL-302-in preclinical development to treat solid cancers.

Tim Hoey, SVP of cancer biology at OncoMed Pharmaceuticals Inc., said it would be useful "to carry out studies combining the Notch-specific antibodies with existing cancer therapies to see if anti-NOTCH1 treatment results in additive or synergistic activity."

OncoMed's OMP-21M18, an antibody targeting Notch ligand delta-like ligand 4 (DLL4), is in Phase I testing to treat advanced solid tumors and is partnered with GlaxoSmithKline plc. In an in vivo tumor growth assay, the antibody alone or in combination with chemotherapy reduced cancer stem cell frequency and decreased rates of breast tumor recurrence compared with chemotherapy alone. ${ }^{9}$

In terms of safety, researchers have developed dosing regimens that are helping circumvent the GI toxicity associated with $\gamma$-secretase inhibitors. "The Nature paper perhaps overstates the GI toxicity 
associated with $\gamma$-secretase inhibitors, as we now know how to better dose those compound to prevent many of the side effects," said Max Wicha, professor of oncology at the University of Michigan Medical School, director of the University of Michigan Comprehensive Cancer Center and a cofounder of OncoMed.

Wicha and colleagues at the Baylor College of Medicine and Dana-Farber Cancer Institute are running a Phase I trial of MK0752, a $\gamma$-secretase inhibitor from Merck \& Co. Inc., in combination with docetaxel to treat advanced breast cancer.

Wicha said that by dosing MK-0752 three days out of every three weeks, he and his colleagues are able to avoid much of the GI toxicity. Also, last year Merck researchers published animal data showing that three days of $>70 \%$ Notch pathway inhibition using a $\gamma$-secretase inhibitor was sufficient to provide an antitumor effect and was well tolerated. ${ }^{10}$

Stemline's Cirrito said another approach to reducing the toxicity of $\gamma$-secretase inhibitors is to combine them with steroids. For example, a group at Columbia University led by Adolfo Ferrando, assistant professor of pathology and pediatrics at the university's medical school, reported last year that combining a $\gamma$-secretase inhibitor with glucocorticoids reduced gut toxicity without impairing efficacy in a mouse model of T-ALL. ${ }^{11}$

"I would really like to see a head-to-head comparison of the Notchspecific antibodies with properly dosed $\gamma$-secretase inhibitors. That would allow us to see if there really are significant efficacy and safety benefits associated with the antibodies," said Wicha.
Fulmer, T. SciBX 3(17); doi:10.1038/scibx.2010.512

Published online April 29, 2010

REFERENCES

1. Wu, Y. et al. Nature; published online April 15, 2010; doi:10.1038/nature08878

Contact: Christian Siebel, Genentech Inc., South San Francisco, Calif. e-mail: csiebel@gene.com

2. Kopan, R. \& llagan, M.X. Cell 137, 216-233 (2009)

3. van Es, J. et al. Nature 435, 959-963 (2005)

4. Deangelo, D. et al. J. Clin. Oncol. 2006 ASCO Annu. Meet. Proc. Part I 24, 6585 (2006)

5. Ferrando, A. Hematology 1, 353-361 (2009)

6. Massi, D. et al. Mod. Pathol. 19, 246-254 (2005)

7. Lee, S. et al. Cancer Sci. 100, 920-926 (2009)

8. Haruki, N. et al. Cancer Res. 65, 3555-3561 (2005)

9. Hoey, T. et al. Cell Stem Cell 5, 168-177 (2009)

10. Tammam, J. et al. Br. J. Pharmacol. 158, 1183-1195 (2009)

11. Real, P. et al. Nat. Med. 15, 50-58 (2009)

COMPANIES AND INSTITUTIONS MENTIONED

Aveo Pharmaceuticals Inc. (NASDAQ:AVEO), Cambridge, Mass. Baylor College of Medicine, Houston, Texas Columbia University, New York, N.Y.

Dana-Farber Cancer Institute, Boston, Mass.

Exelixis Inc. (NASDAQ:EXEL), South San Francisco, Calif. Genentech Inc., South San Francisco, Calif. GlaxoSmithKline plc (LSE:GSK; NYSE:GSK), London, U.K. Harvard Medical School, Boston, Mass.

Merck \& Co. Inc. (NYSE:MRK), Whitehouse Station, N.J.

OncoMed Pharmaceuticals Inc., Redwood City, Calif. Roche (SIX:ROG; OTCQX:RHHBY), Basel, Switzerland Stemline Therapeutics Inc., New York, N.Y. University of Michigan Comprehensive Cancer Center, Ann Arbor, Mich.

University of Michigan Medical School, Ann Arbor, Mich. 